\title{
Is a description deeper than the quantum one possible?
}

\author{
GianCarlo Ghirardi ${ }^{1}$ and Raffaele Romano ${ }^{2}$ \\ ${ }^{1}$ Department of Physics, University of Trieste, the Abdus Salam ICTP, Trieste, Italy \\ E-mail: ghirardi@ictp.it \\ ${ }^{2}$ Department of Mathematics, Iowa State University, Ames IA, USA \\ E-mail: rromano@iastate.edu
}

\begin{abstract}
.
Recently, it has been argued that quantum mechanics is a complete theory, and that different quantum states do necessarily correspond to different elements of reality, under the assumptions that quantum mechanics is correct and that measurement settings can be freely chosen. In this work, we prove that this result is a consequence of an unnecessarily strong mathematical expression of the free choice assumption, which embodies more conditions than explicitly stated. The issues of the completeness of quantum mechanics, and of the interpretation of the state vector, are by no means resolved. Taking this perspective, we describe how the recently introduced class of crypto-nonlocal hidden variables theories can be used to characterize the maximal possible departure from quantum mechanics, when the system consists of a pair of qubits.
\end{abstract}

PACS numbers: 03.65.Ta, 03.65.Ud 


\section{Introduction}

Despite the unprecedented success of quantum theory (and its field-theoretical relativistic generalization) in explaining any experimental evidence of the microscopic world, the interpretation of the formalism represents a long-standing problem. This is partially due to the counter-intuitive features of the description of the micro-world, when compared to the concepts derived from classical physics, the prominent examples being given by probabilism, indeterminism and non-locality. Nonetheless, the really unpleasant feature of the quantum formalism is the fact that it appears more like a set of operational prescriptions to fit the experimental data, rather than a coherent description of reality. The theory relies on two different kinds of evolution depending on the rather vague notion of measurement, is not able to account for the behavior of the classical world in the limit of macroscopic objects, and, consequently, its range of validity is not well defined.

The famous incompleteness argument by Einstein, Podolski and Rosen has raised the questions of whether quantum mechanics is a complete theory or not, and how to interpret the state description required by the theory in terms of the quantum state vector $\psi$. In particular, it is unclear whether $\psi$ represents a state of reality or rather a state of knowledge, as suggested by its updating following a measurement procedure. According to these lines, several ontological models of quantum mechanics have been introduced, that is, theories which are predictively equivalent to quantum mechanics, but providing a possibly richer description of the microscopic reality through the socalled ontic state, the most accurate specification of the physical state of the system, at least in principle. In these theories, the state vector $\psi$ might embody only partial information on the ontic state, since it is associated to a distribution $\rho_{\psi}(\lambda)$ on the space of the ontic variable $\lambda$, with $\rho_{\psi}(\lambda) \geq 0$ and

$$
\int \rho_{\psi}(\lambda) d \lambda=1 \quad \text { for all } \psi \text {. }
$$

The ontic state $\lambda$ accounts for the elements of reality of the underlying theory since it provides the complete description of the state of the system, but, in principle, it might be not fully accessible. This is the reason why these models were formerly characterized as hidden variables theories.

Notice that the ontological status of $\psi$ can be understood by studying the distribution $\rho_{\psi}(\lambda)$, which, in the so-called $\psi$-epistemic models, has overlapping supports,

while in the so-called $\psi$-ontic models, it has disjoint supports [1] for different $\psi$. Accordingly, in a $\psi$-ontic theory different state vectors necessarily correspond to different ontic states, whereas in a $\psi$-epistemic theory they could correspond to the same ontic state. One could associate to $\psi$ well defined elements of reality only in the first case.

In a recent work it has been argued that, if quantum mechanics is correct and the experimenters can freely choose their own settings, no theory can outperform its predictive power, that is, the microscopic world can only be described in terms of probabilistic laws, and the probabilities are definitely those provided by the quantum 
formalism [2]. In other words, quantum mechanics really is complete. Moreover, as a corollary of this result, it has been proven that $\psi$-epistemic models necessarily contrast with quantum mechanics [3]. Accordingly, it has been concluded that $\psi$ does not represents a state of knowledge but rather a state of reality. This result already appeared in the recent literature [4], although limited by the assumption that factorized quantum states correspond to factorized states of the underlying theory, as highlighted by an explicit model [5].

In our opinion, the general scenario envisaged in [2, 3] is not the right one, since the mathematical expression of the free choice assumption, denoted by $F R$, is unnecessarily strong. While a general criticism to these works from a more epistemological perspective has been presented in Ref. [6], here we prove that $F R$ embodies more than the free choice assumption. Therefore, we provide evidence that the argument put forward in [2, 3] is not conclusive for stating the completeness of quantum mechanics, nor in determining the ontological status of the quantum state vector.

This result triggers the following question: are there theories which are compatible with quantum mechanics, but potentially distinguishable from it? We argue that this question has a positive answer, and the theories fulfilling this requirement are exactly the recently introduced crypto-nonlocal hidden variables models. We discuss what is the maximal departure from quantum expectations that these models can provide in the simple case of a pair of two-level systems.

\section{Free will and the ontological status of quantum mechanics}

As already anticipated, Ref. [2] derives completeness of quantum mechanics and the one-to-one correspondence between quantum state vectors and elements of reality from the assumptions $Q M$ of the validity of the predictions of quantum mechanics, and a request $F R$ which, according to the authors, expresses the freedom of choosing the measurement settings. One considers two space-like separated observers performing local measurements on the two parties of an entangled state $\psi$. The measurement settings are given by vectors $A$ and $B$, the outcomes are denoted by $X$ and $Y$. Following [2, 3], we assume that additional information on the ontic state $\lambda$ is available and can be obtained through a measurement with setting $C$ and output $Z$. In the following, we do not exclude the case $Z=\lambda$, which means that the ontic state is fully accessible. We consider all these quantities as random variables. The $F R$ assumption is the condition that

[...] the input $A$ can be chosen to be uncorrelated with all the space-time random variables whose coordinates lie outside the future light-cone of its coordinates [2],

and the same requirement holds also for $B$ and $C$. The authors of [2] have expressed this assumption by imposing the following constraints on the conditional probabilities:

$$
P_{A \mid B C Y Z}=P_{A}, \quad P_{B \mid A C X Z}=P_{B}, \quad P_{C \mid A B X Y}=P_{C},
$$


which are all needed to derive the main results of [2, 3]. However, we notice that the free will condition is consistent, among others, with a condition weaker than $F R$, which makes reference exclusively to the fact that the two observers can independently choose which observables to measure:

$$
P_{A \mid B \lambda}=P_{A}, \quad P_{B \mid A \lambda}=P_{B},
$$

where $\lambda$ is the aforementioned ontic state. This condition, denoted by $F W$ in the following, produces the relevant factorization $P_{A B \lambda}=P_{A} P_{B} P_{\lambda}$. Meaningfully, $F W$ is unrelated with the physically important assumption that the two observers cannot communicate superluminally, denoted as $N S$, and expressed by

$$
P_{X \mid A B}=P_{X \mid A}, \quad P_{Y \mid A B}=P_{Y \mid B}
$$

This is reasonable: one could imagine artificial models in which free will and superluminal signalling coexist. Notice that $F R \Rightarrow N S$, supporting the idea that $F R$ embodies more than the free choice assumption.

We observe that in [2] it is pointed out that the information supplementing $\psi$

[...] must be static, that is, its behavior cannot depend on where or when it is observed.

Otherwise said, the region of events corresponding to the acquisition of this information can be chosen to be space-like with respect to the events associated to $A$ and $B$. In [2], this statement is presented as a simple remark and it does not constitute a new assumption; nonetheless, here we choose to denote it by $S T$, and to express it as $P_{C Z \mid A B X Y}=P_{C Z}$. It turns out that

$$
F W \wedge N S \wedge S T \Rightarrow F R \text {. }
$$

In fact, from $S T$ it follows that $P_{A B Y \mid C Z}=P_{A B Y}$; moreover we have

$$
P_{A B Y \mid C Z}=P_{A \mid B Y C Z} P_{B Y \mid C Z}=P_{A \mid B Y C Z} P_{B Y}
$$

by using again $S T$, but also

$$
P_{A B Y}=P_{A B} P_{Y \mid A B}=P_{A} P_{B} P_{Y \mid B}=P_{A} P_{B Y}
$$

from $N S$ and $F W$. By comparing ([6) and (7) we find that $P_{A \mid B Y C Z}=P_{A}$, and a similar argument proves that $P_{B \mid A X C Z}=P_{B}$. Finally, $P_{C \mid A X B Y}=P_{C}$ is a direct implication of $S T$. This result clearly shows that $F R$ corresponds to more than the free will of the observers. Notice that, if we assume $Z=\lambda$, we can prove also the converse of implication (5), meaning that, if the ontic state would be completely accessible, $F R$ would be equivalent to the conjunction of the conditions $F W, N S$ and $S T$. For a detailed account of the role of the accessibility of $\lambda$ in this analysis, see [6].

Therefore, violation of $F R$ does not necessarily imply lack of free will as long as $S T$ or $N S$ are violated. This means that ontological models fully consistent with quantum mechanics, with the free will assumption (expressed through $F W$ ) and without superluminal communication are indeed possible, as long as the supplementary 
information on the ontic state is not static. Moreover, these models could be made of the $\psi$-epistemic type, for instance, by following the lines described in [5].

We want to comment about our condition $F W$. We do not consider it as the ultimate expression of the free will assumption, but only a meaningful substitute to $F R$, which enables us to raise our criticism to the $F R$ assumption. As $F R$, also $F W$ relies on conditional probabilities, and, in our opinion, this is not the most appropriate way to express the free will. Our target here is only to prove that the conclusions of [2, 3] are not appropriate, rather than providing an accurate mathematical expression of the free will assumption. Notice that the general approach to free will has been expressed by J.S. Bell as

for me this means that the values of such variables have implications only in their future light cones [7],

and, in our opinion, neither $F R$ nor $F W$ are able to properly express this fact, since lack of correlations is stronger than lack of implications.

\section{Beyond quantum mechanics: the role of crypto-nonlocal hidden variables models}

Following our reasoning, we conclude that the issue about completeness of quantum mechanics is still open, and similarly there are not conclusive conclusions concerning the ontological status of the vector $\psi$, which represents the state of the system in quantum mechanics. We now focus on the following question: could there be a theory, predictively equivalent to quantum mechanics, but experimentally distinguishable from it? In other words, would it be possible that a more refined knowledge of $\lambda$ could produce different outcomes (e.g. different statistics) from quantum mechanics, consistently with the fact that, if the information on the state reduces to that encoded in $\psi$, these outcomes are exactly those of quantum mechanics? Of course, one has to further constrain the theory in order to avoid physical inconsistencies. More explicitly, any information on $\lambda$ cannot be used to implement superluminal communication between distant parties.

It turns out that this requirement is exactly addressed by the class of cryptononlocal hidden variable models, recently introduced by Leggett in a different context (investigation of non-locality and entanglement of correlated photons) [8]. In the simplified case where the ontic state, jointly with the settings $a$ and $b$ (the actual values of the random variables $A$ and $B$ ) determine the outcomes $x$ and $y$ (the actual values of the random variables $X$ and $Y$ ) $\ddagger$, these models can be described as follows. We express $\lambda$ through two variables $(\mu, \tau), \mu$ denoting the unaccessible part of the ontic state, and $\tau$ the accessible one. Now, we can write $\rho_{\psi}(\lambda)=\rho_{\psi, \tau}(\mu) \rho_{\psi}(\tau)$, and impose that knowledge of $\tau$ does not allow superluminal communication, that is

$$
\int x(a, b, \lambda) \rho_{\psi, \tau}(\mu) d \mu=f_{\psi}(a, \tau),
$$

$\ddagger$ In general, only the probabilities of these outcomes are determined. 


$$
\int y(a, b, \lambda) \rho_{\psi, \tau}(\mu) d \mu=g_{\psi}(b, \tau),
$$

which are the so-called non-signalling conditions. The quantities $f_{\psi}(a, \tau)$ and $g_{\psi}(b, \tau)$ are the local averages of the theory at the intermediate level, that is, when the state of the system is described by $\tau$. Non-locality, which is apparent by the functional dependence $x(a, b, \lambda)$ and $y(a, b, \lambda)$, has been canceled out. As required, when we additionally average over $\tau$ we recover the quantum expectations,

$$
\begin{aligned}
\int f_{\psi}(a, \tau) \rho_{\psi}(\tau) d \tau & =\langle x(a)\rangle_{\psi}, \\
\int g_{\psi}(b, \tau) \rho_{\psi}(\tau) d \tau & =\langle y(b)\rangle_{\psi} .
\end{aligned}
$$

The theory is experimentally distinguishable from quantum mechanics as long as $f_{\psi}(a, \tau) \neq\langle x(a)\rangle_{\psi}$ and $/$ or $g_{\psi}(b, \tau) \neq\langle y(b)\rangle_{\psi}$.

An example of this scheme has been recently described in [9] for a pair of two qubits. It is a generalization of the famous Bell's model for the singlet state of a pair of two-level systems, valid for an arbitrary state $\psi$ written as

$$
|\psi\rangle=\sin \frac{\theta}{2}|00\rangle+\cos \frac{\theta}{2}|11\rangle,
$$

with $\theta \in[0, \pi / 2]$. If $\theta=0,|\psi\rangle$ is a separable state state; if $\theta=\pi / 2$ it is a maximally entangled state. The ontic state is given by the pair $\lambda=(\psi, \tilde{\lambda})$, where $\tilde{\lambda}$ is a unit vector in the 3-dimensional real space. By construction, the model is $\psi$-ontic, because different vectors $\psi$ are necessarily associated to different ontic states $\lambda$ \& . For specific values of the local settings, $A=a$ and $B=b$ ( $a$ and $b$ are real, unit vectors), the local observables are given by $\sigma \cdot a$ and $\sigma \cdot b$, where $\sigma=\left(\sigma_{x}, \sigma_{y}, \sigma_{z}\right)$ is the vector of Pauli matrices. In particular, $\sigma_{z}$ is defined so that $|0\rangle$ and $|1\rangle$ are its +1 and -1 eigenvectors respectively. Without loss of generality we assume that $a$ and $b$ lie in the plane orthogonal to the direction of propagation of the entangled particles, assumed to depart from a common source. The local measurement outcomes are given by

$$
x(a, b, \tilde{\lambda})= \begin{cases}+1, & \text { if } \hat{a} \cdot \tilde{\lambda} \geq \cos \xi, \\ -1, & \text { if } \hat{a} \cdot \tilde{\lambda}<\cos \xi,\end{cases}
$$

and

$$
y(b, \tilde{\lambda})= \begin{cases}+1, & \text { if } b \cdot \tilde{\lambda} \geq \cos \chi \\ -1, & \text { if } b \cdot \tilde{\lambda}<\cos \chi\end{cases}
$$

In the previous relations, $\hat{a}=\hat{a}(a, b)$ is in the plane of $a$ and $b$, as detailed in [9]; moreover, $\cos \xi=-\langle x(a)\rangle_{\psi}$, and $\cos \chi=-\langle y(b)\rangle_{\psi}$.

With the additional assumption that $\tilde{\lambda}$ is uniformly distributed on the unit sphere, it is possible to prove that this model is predictively equivalent to quantum mechanics (see 9 for the details). Moreover, it belongs to the crypto-nonlocal family. We identify $(\mu, \tau)$ with the spherical coordinates of $\tilde{\lambda}: \mu$ is the azimuthal angle and $\tau$ the polar angle, and the north pole is identified by the direction of the incoming particle. It is easy to $\S$ Nonetheless, a $\psi$-epistemic model can be obtained by suitably modifying this scheme. 


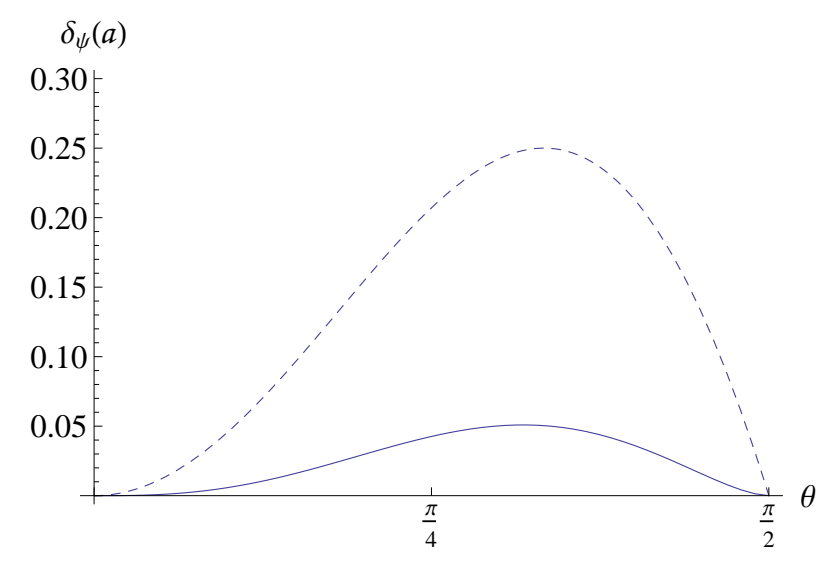

Figure 1. Behavior of $\delta_{\psi}(a)$ as a function of $\theta$. Solid line: $\delta_{\psi}(a)$ for the generalization of the Bell's model described in the text; dashed line: upper bound for $\delta_{\psi}(a)$. In both cases we consider the particular choice $a=(0,0,1)$.

prove that $\rho_{\psi, \tau}(\mu)=1 / 2 \pi$, and, by construction, integration over $\mu$ cancels non-locality in local averages. We find that 9 ]

$$
f_{\psi}(a, \tau)=\frac{1}{\pi} \cos ^{-1}\left(\frac{2\langle x(a)\rangle_{\psi}^{2}}{\sin ^{2} \tau}-1\right)-1,
$$

if $\left|\tau-\frac{\pi}{2}\right| \leq \xi$ and $f_{\psi}(a, \tau)=-1$ otherwise, and a similar relation (with $\xi$ replaced by $\chi)$ for $g_{\psi}(b, \tau)$. In general, $f_{\psi}(a, \tau) \neq\langle x(a)\rangle_{\psi}$ and $g_{\psi}(b, \tau) \neq\langle y(b)\rangle_{\psi}$. Therefore, despite the model is absolutely artificial, it provides evidence that models compatible with quantum mechanics, but in principle distinguishable from it, are indeed possible, without violating the free will assumption.

In [10, as a measure of the maximal departure from quantum expectations that crypto-nonlocal hidden variables models models can provide, we have used the variance of the variable $f_{\psi}(a, \tau)$ over the distribution $\rho_{\psi}(\tau)$ :

$$
\delta_{\psi}(a)=\int\left(f_{\psi}(a, \tau)-\langle x(a)\rangle_{\psi}\right)^{2} \rho_{\psi}(\tau) d \tau .
$$

When the system consist of a pair of qubits, we have expressed the upper bound for this quantity for generic models as

$$
\delta_{\psi}(a) \leq \cos \theta-\langle x(a)\rangle_{\psi}^{2} .
$$

In Fig. 1 we show the dependence of this constraint by entanglement, and plot the corresponding curve for the specific model described in [9].

\section{Final remarks and conclusions}

In this contribution we have criticized the form of the free will assumption which has been recently adopted to derive some striking results, in particular that (i) quantum mechanics is a complete theory, and (ii) the quantum state vector $\psi$ is necessarily in one-to-one correspondence with the elements of reality of the theory. To strengthen 
our argument, we have provided a different definition of free will, which makes clear that the former free will assumption actually encodes more than the observers' free choice (that is, the condition that the theory is non-signalling, and the staticity of the information supplementing $\psi$ ). We believe that both approaches to free will do not represent the correct necessary and sufficient condition for this assumption, and we conjecture that this condition cannot be simply expressed through simple expressions involving conditional probabilities.

Therefore, ontological models which are compatible but possibly experimentally distinguishable from quantum mechanics are possible. Since these models should necessarily be non-signalling, we have suggested that they are given by the class of crypto-nonlocal hidden variables theories, and we have provided a simple example. Finally, we have described an upper bound for the local averages of any deterministic ontological theory for quantum mechanics in the case a pair of qubits. These results

suggest that crypto-nonlocal hidden variables theories represent a relevant tool in the study of non-locality.

\section{Acknowledgments}

This research is partially supported by the ARO MURI grant W911NF-11-1-0268.

\section{References}

[1] N. Harrigan and R.W. Spekkens, Found. Phys. 40, 125 (2010)

[2] R. Colbeck and R. Renner, Nature Comm. 2, 411 (2011)

[3] R. Colbeck and R. Renner, Phys. Rev. Lett. 108, 150402 (2012)

[4] M.F. Pusey, J. Barrett and T. Rudolph, Nature Phys. 8, 476 (2012)

[5] P.G. Lewis, D. Jennings, J. Barrett and T. Rudolph, Phys. Rev. Lett. 109, 150404 (2012)

[6] G.C. Ghirardi and R. Romano, Foundations of Physics: Volume 43, Issue 7881 (2013)

[7] J.S. Bell, Free variables and local causality, in Speakable and unspeakable in quantum mechanics, Cambridge University Press (1987)

[8] A.J. Leggett, Found. Phys. 33, 1469 (2003)

[9] G.C. Ghirardi and R. Romano, Phys. Rev. A 85, 042102 (2012)

[10] G.C. Ghirardi and R. Romano, Phys. Rev. Lett. 110, 170404 (2013) 